\title{
Isolation of candida species from the oral cavity and fingertips of complete and partial dentures wearers
}

\begin{abstract}
Purpose: Removable denture wearers commonly use their fingers to take out their prostheses from their mouths and oral Candida, if present, may colonize their fingers and constitute a source of contamination. The aim of this study was to find out the difference in oral and fingertips Candida isolation before and after wearing the prostheses, and to investigate the association between oral and fingertip Candida colonization.

Methods: Fifty healthy subjects, wearing removable complete or partial dentures for the first time were included. Oral Candida species were isolated using the concentrated oral rinse technique while fingertips Candida were isolated by pressing the fingers of both hands on a plate of Sabouraud's dextrose agar. Candida species were identified using the VITEK $2 ®$ yeast identification system. Three samples were taken from each subject; one at the insertion of the prosthesis, after one month and after two months of the insertion visit.

Results: The prevalence and density of oral Candida colonization were both higher after one and two months of the insertion of removable complete and partial dentures but the difference was not statistically significant $(\mathrm{P}>0.05)$. No statistically significant difference was also found between the complete denture wearers (CDW's) and partial denture wearers (PDW's) regarding the mean candidal count $(\mathrm{P}>0.05)$. Oral and fingertips Candida species presence among the same CDWs or PDWs at the initial, first and second visits was statistically insignificant.
\end{abstract}

Conclusion: There was no association between oral and fingertips Candida species carriage among healthy CDWs or PDWs.
Volume I Issue 3 - 2014

\author{
Ziad Nawaf Al Dwairi,' Azmi Mohammad \\ Ghaleb Darwazeh, ${ }^{2}$ Lubabah Abdul Kader \\ Shukri' \\ 'Department of Prosthodontics, Faculty of Dentistry, Jordan \\ University of Science and Technology, Jordan \\ ${ }^{2}$ Department of Oral Medicine and Surgery, Faculty of Dentistry, \\ Jordan University of Science and Technology, Jordan
}

\begin{abstract}
Correspondence: Ziad Nawaf Al-Dwairi, Department of Prosthodontics, Faculty of Dentistry, Jordan University of Science and Technology, Po Box 3030, code 221 10, Irbid, Jordan, Email ziadd@just.edu.jo
\end{abstract}

Received:June 10,2014 | Published: June 18, 2014

\section{Introduction}

Candida species constitute part of the oral commensal flora in about $20-60 \%$ of the population. ${ }^{1}$ Oral carriage of Candida organisms without any noticed signs or symptoms had been recognized for many years ${ }^{2}$ and the posterior tongue is considered to be its primary location. In addition to oral mucosa, the yeast can also colonize dental plaque. ${ }^{3}$ Candida albicans can become virulent and responsible for oral candidiasis depending on the local and systemic factors related principally to the host conditions. ${ }^{4}$ Several factors will dictate the colonization of $C$. albicans in the oral cavity, their quantity and pathogenicity. Nutritional disorders, diabetes mellitus, long-term antibiotic or corticosteroid use, head and neck cancer, radiation therapy and AIDS are considered important predisposing factors. ${ }^{4,5}$ It has been shown that dentures can facilitate oral Candida carriage and predispose their wearers to the onset of oral candidiasis. Both poor oral and poor denture hygiene, with plaque accumulated on the denture, contribute to the emergence of oral candidiasis. ${ }^{5}$

Candida species have been shown to contaminate the hands of hospital personnel ${ }^{6}$ and have been isolated simultaneously from the mouth and hands of some AIDS patients ${ }^{7}$. Removable denture wearers use their fingers frequently to take out their prostheses from their mouths and theoretically oral Candida, if present, may contaminate their fingers. The Candida-contaminated fingers could consequently constitute a source of contamination or/and reinfection. Only one study investigated the association between oral and fingertip Candida isolation in a group of complete denture wearers and found that the hands of patients who had oral candidaisis were significantly more colonized with Candida species than oral Candida-free subjects. ${ }^{8}$
The aforementioned study investigated the association between oral and fingertip Candida colonization in subjects with denture associated oral candidiasis. Up to the authors' knowledge, no previous studies investigated the significance of hand Candida as a source for mouth infection and re-infection in healthy patients wearing removable prosthesis. Therefore, the aim of this study is to find out if there are differences in oral and fingertips Candida isolation before and after wearing removable complete or partial dentures and to find out any possible association between oral and fingertip Candidacolonization.

\section{Material and methods}

\section{Study sample}

A total of 50 subjects ( 25 completely edentulous and 25 partially edentulous) were recruited from the Removable Prosthodontics Clinic at the Dental Teaching Center/Jordan University of Science and Technology located in the North of Jordan and enrolled in the study after obtaining the necessary ethical approval.

Inclusion criteria included apparently healthy subjects who:

1. Reported the absence of any medical history that predispose them to oral candidiasis, or can promote Candida carriage (such as diabetes mellitus, anemia, and immune-debilitating disease).

2. Had not used any removable prosthesis before.

3. Had not used any antifungal agents or antiseptic mouthwashes over the past six months.

4. Had not used any medication known to predispose to oral candidiasis, e.g. antibiotic or steroid therapy over the last six months. 
Clinical examination confirmed that none of the included subjects had signs or symptoms suggestive of oral candidiasis, candidal paronychia or dermatologic fungal infection. Twenty five subjects were provided with removable acrylic complete dentures and 25 others with partial dentures. All 50 dentures were constructed by undergraduate dental students or by the internship dentists at the Dental Teaching Center following standardized clinical techniques under the supervision of the academic faculty members in the Department of Prosthodontics. All dentures were processed by dental technicians within the same institution.

For each subject, six microbiologic samples were taken in total over three visits; one pair (mouth rinse and fingerprints) in each visit with an interval period of one month between the visits. Clinical oral examination was carried out in each visit.

The subject was considered as a smoker if he/she had smoked at least ten cigarettes per day for the past year, while a subject who never had smoked or had stopped smoking for at least one year was considered to be a non-smoker. ${ }^{9}$

At the denture insertion visit (initial visit), dentures were checked and approved by a prosthodontist. All subjects were provided with routine verbal and written denture hygiene instructions. To attempt to standardize some of the effect of diurnal variation, meal eating and tooth brushing, ${ }^{10}$ samples were always taken between 10:00 am and 3:00 $\mathrm{pm}$ and processed on the same day.

The concentrated oral rinse technique was used for oral Candida isolation. ${ }^{11}$ The subject was provided with $10 \mathrm{~mL}$ of sterile phosphate-buffered saline (PBS $0.01 \mathrm{M}, \mathrm{pH}$ 7.2) in a sterile disposable universal container and asked to rinse the mouth for a period of 60 seconds while not wearing any denture. After that, the rinse was expectorated back into the same container. Samples were then transported immediately to the microbiology laboratory at the King Abdullah University Hospital for microbiological processing and assessments.

Fingertip Candida sampling was performed by asking each subject to press gently using the fingertips of both hands for one minute each on a plate of Sabouraud's dextrose agar (Oxoid Ltd, Basingstoke, Hampshire, England). ${ }^{8}$

One month after the initial visit (1st visit), information about each subject's denture hygiene frequency, and hand-washing habits after denture handling was obtained through a structured interview conducted immediately before the samples were taken.

According to the method of Ambjornsen et al., ${ }^{12}$ the denture plaque index was scored in representative areas of the fitting surface of the complete and partial dentures. The individual scores were added and the sum was divided by the number of areas. Denture plaque scoring was carried out by one investigator who was calibrated before the start of the study.

Two months after the initial visit (2nd visit), the same sampling techniques and questions implemented in the first visit were employed.

Isolation, quantification and identification of Candida species The oral rinse sample was centrifuged at $3000 \mathrm{rpm}$ (Jouan C412, France) for 10 minutes. The supernatant was discarded and one $\mathrm{mL}$ of sterile PBS was added to the deposit and agitated on a bench vibrator (VibroFix VF1 electronic, Italy) for 30 seconds for disaggregation of microorganisms. Afterwards, $0.5 \mathrm{~mL}$ of the processed mixture was inoculated on a plate of Sabouraud's dextrose agar (Oxoid Ltd, Basingstoke, Hampshire, England). The plates of fingerprint and mouth rinse samples were then incubated aerobically at $37^{\circ} \mathrm{C}$ for 48 hours and then at room temperature for another 24 hours for optimal fungal growth. Candida colonies were identified based on the colony color, texture and morphology, and determination of the purity of the culture was then performed microscopically by the wet mount technique using the X40 objective. The number of colonies on the plates of oral rinse cultures was quantified and multiplied by two to work out the number of colony forming units per $\mathrm{ml}$ of the rinse $(\mathrm{CFU} / \mathrm{ml})^{11}$. All the Candida isolates were further identified based on their carbohydrate assimilation pattern by the VITEK $2{ }^{\circledR}$ yeast identification system (bioMérieux, Craponne, France). ${ }^{13}$

\section{Statistical analyses}

The results were analyzed by using the Statistical Package for Social Sciences (SPSS) software version 11.0 (SPSS $®$ : Inc., Chicago, IL, USA). Kappa statistics and McNemar tests were produced to test the agreement and differences between matched readings. The Pearson chi-square test, Fisher exact test, Mann-Whitney and one way ANOVA tests were performed for comparisons among sub-groups. The level of significance was set at $\mathrm{P} \leq 0.05$.

\section{Results}

Of the 50 subjects, 25 were provided for the first time with maxillary and mandibular complete dentures, and other 25 provided for the first time with partial dentures (11 maxillary dentures, 9 mandibular dentures, and 5 cases with both maxillary and mandibular partial dentures. The mean age $( \pm \mathrm{SD})$ of the study groups were $52.6 \pm 10.34$ years for the complete denture wearers (CDW's) and 45.6 \pm 12.66 years for the partial denture wearers (PDW's). Candida carriage was not significantly different between age groups, genders or smokers and non-smokers (Table 1). As shown in Table 2, there was a trend to an increase in the oral and fingertip Candida carriage rate over the 3 visits although the increase was not statistically significant.

At the initial visit, none of the subjects had a simultaneous positive oral and fingertips Candidaisolation, while $3(6.0 \%)$ subjects at the first visit had positive oral and fingertips Candida species, 2 of them were PDW's and one was a CDW. At the second visit, only two PDW's were found to be carriers of Candida species in their mouths and fingerprints (Table 3). Oral and fingertips Candida species presence among the same CDW or PDW at the initial, first and second visits was statistically not significant (Kappa $=0.042, \mathrm{P}=0.384$ ).

The mean count of oral Candida species generally increased over the three visits, but the increase was statistically not significant (Table 4). Candida albicans, C. dubliniensis and C. parapsilosis were the three Candida species isolated from the fingertips samples during the three visits (Table 5).

Three subjects of the group who had reported to not wash their hands after denture handling were carriers of Candida species at their fingertips at the first visit, and just one subject a was carrier at the second visit $(\mathrm{P}=0.608)$. There was a significant correlation between not washing hands and increased oral Candida carriage; however the relationship with fingertip Candida carriage was not significant (Table $6)$. 
Table I Isolation of oral Candida species in relation to age, gender, and smoking habits among the study subjects ( $N=50)$

\begin{tabular}{|c|c|c|c|}
\hline & \multicolumn{3}{|l|}{ Candida Isolation } \\
\hline & No. of Subjects (\%) & P -Value* & CFU/mL $\pm S D$ \\
\hline Age (Years) & & 0.064 & \\
\hline$\leq 40(n=12)$ & $9(75.0)$ & & $73.56 \pm 93.4$ \\
\hline $41-65(n=33)$ & $14(42.4)$ & & $|3| .72 \pm 228.3$ \\
\hline$>65(n=5)$ & $I(20.0)$ & & $60.0 \pm 0.0$ \\
\hline Gender & & 0.423 & \\
\hline Male $(n=32)$ & $14(43.8)$ & & $104.0 \pm 202.32$ \\
\hline Female $(n=18)$ & $10(55.6)$ & & $111.0 \pm 161.74$ \\
\hline Smoking & & 0.272 & \\
\hline Yes $(n=17)$ & $10(58.8)$ & & $28.6 \pm 28.2$ \\
\hline No $(n=33)$ & I4(42.4) & & $162.8 \pm 224.6$ \\
\hline
\end{tabular}

*Pearson Chi-squre test or Fisher's exact test, **Mann-Whitney test, one way-ANOVA

Table 2 Isolation of Candida species from oral cavity and fingertips samples during the three visits $(\mathrm{N}=50)$

\begin{tabular}{|c|c|c|c|c|c|c|c|}
\hline \multicolumn{3}{|c|}{ Oral Candida Isolation } & \multirow{3}{*}{$\begin{array}{l}\text { P -Value } \\
\left(\chi^{2}-\text { Test }\right)\end{array}$} & \multicolumn{3}{|c|}{ Finger Tips Candida Isolation } & \multirow{3}{*}{$\begin{array}{l}\text { P -Value } \\
\left(\chi^{2}-\text { Test }\right)\end{array}$} \\
\hline Initial Visit & I $^{\text {st }}$ Visit & $2^{\text {nd }}$ Visit & & Initial Visit & Ist $^{\text {st }}$ isit & $2^{\text {nd }}$ Visit & \\
\hline$N(\%)$ & $N(\%)$ & $N(\%)$ & & $N(\%)$ & $N(\%)$ & $N(\%)$ & \\
\hline $24(48.0)$ & $25(50.0)$ & $27(54.0)$ & 0.830 & $2(4.0)$ & $3(6.0)$ & $4(8.0)$ & 0.701 \\
\hline
\end{tabular}

Table 3 The relationship between oral and fingertip Candida species isolation according to denture status during the three visits $(\mathrm{N}=50)$

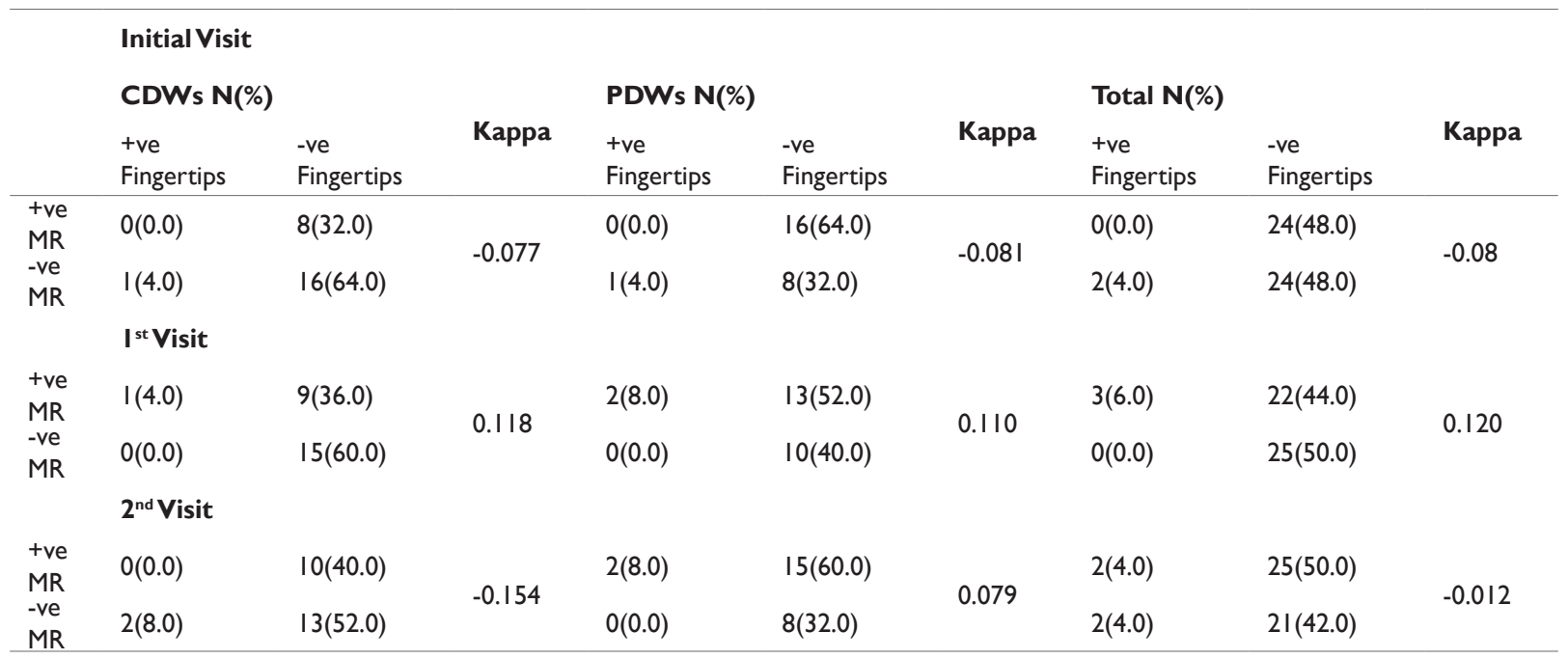

MR: Mouth Rinse

Table 4 The mean count of Candida species (SD) isolated from the oral rinse samples of CDWs (N=25) and PDWs (N=25) during the three visits

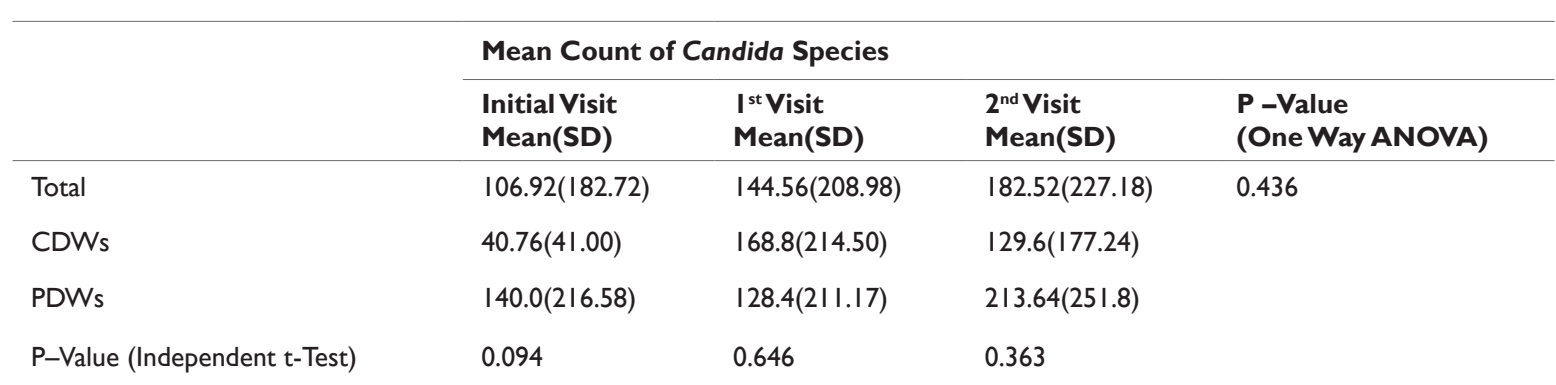

Citation: Dwairi ZNA, Darwazeh AMG, Shukri LAK. Isolation of candida species from the oral cavity and fingertips of complete and partial dentures wearers. J Dent Health Oral Disord Ther. 20I4; I(3):6I-66. DOI: 10.15406/jdhodt.2014.0I.000I5 
As shown in Table 7, the frequency of denture cleansing has no significant effect on the oral Candida carriage. However, there was a statistically significant relationship between the denture plaque index and oral Candida carriage and density $(\mathrm{P}=0.000)$.

Table 5 Candida species isolated from oral rinse samples of the whole subjects during the three visits $(N=50)$

\begin{tabular}{llll}
\hline Candida species & Visits & & \\
\hline C. albicans & 10 & I $^{\text {st }}$ Visit Visit & $2^{\text {nd }}$ Visit \\
C. sphaerica & 2 & 14 & I I \\
C. kefyr & 3 & 1 & 1 \\
C. krusei & 1 & 2 & 5 \\
C. glabarata & 2 & 1 & 0 \\
C. parapsilosis & 0 & 4 & 2 \\
C. tropicalis & 0 & 0 & 0 \\
C. famata & 0 & 0 & 1 \\
C. lusitanie & 1 & 0 & 2 \\
C. dubliniensis & 5 & 0 & 0 \\
\hline
\end{tabular}

Table 6 Relationship of oral and fingertips Candida species with hand washing at the first and second visits $(\mathrm{N}=50)$

\begin{tabular}{|c|c|c|c|c|c|}
\hline & \multicolumn{4}{|c|}{ Hand Wash } & \multirow{3}{*}{$\begin{array}{l}\text { P-Value } \\
\text { McNemar Test }\end{array}$} \\
\hline & \multicolumn{2}{|l|}{ | $^{\text {st }}$ visit } & \multicolumn{2}{|l|}{$2^{\text {nd }}$ visit } & \\
\hline & $\begin{array}{l}\text { No } \\
\mathrm{N}(\%)\end{array}$ & $\begin{array}{l}\text { Yes } \\
N(\%)\end{array}$ & $\begin{array}{l}\text { No } \\
\mathrm{N}(\%)\end{array}$ & $\begin{array}{l}\text { Yes } \\
N(\%)\end{array}$ & \\
\hline Oral Candida carriage & 16(59.3) & $9(39.1)$ & $18(66.7)$ & $9(39.1)$ & 0.000 \\
\hline Fingertips Candida carriage & $3(I I . I)$ & $0(0.0)$ & $\mathrm{I}(3.7)$ & $3(13.0)$ & 0.608 \\
\hline
\end{tabular}

Table 7 The association between oral Candida species carriage and denture plaque and hygiene frequency for the whole sample $(\mathrm{N}=50)$.

\begin{tabular}{llll}
\hline Candida Isolation & & & \\
& No. of Subjects (\%) & P -Value* & CFU/mL \pm SD \\
\hline Denture Cleansing (Time /Day) & & 0.710 & \\
$\leq I(n=18)$ & $9(50.0)$ & & $298.6 \pm 306.6$ \\
Two $(n=16)$ & $10(62.5)$ & $84.2 \pm 104.9$ \\
Three $(n=16)$ & $8(50.0)$ & 0.000 & $174.8 \pm 197.2$ \\
Plaque Index & & & \\
$0(n=15)$ & $0(0.0)$ & & $0 \pm 0.0$ \\
$I(n=35)$ & $27(77.1)$ & $182.52 \pm 227.2$ \\
\hline
\end{tabular}

*Pearson Chi-squre test or Fisher's exact test, **Mann-Whitney test, one way-ANOVA, ***NA: not applicable

\section{Discussion}

The removable dental prosthesis has been frequently considered as an important factor that affects the oral carriage and infection with Candida. ${ }^{14}$ Since previous studies have shown that Candida species are more common in the oral cavity of diabetic or anemic patients than in those of healthy individuals, ${ }^{15,16}$ this study included only apparently healthy subjects who would then be wearing a removal dental prosthesis for the first time. The number of females who participated in this study was less than that of males; however, the results showed that females were more frequent carriers of Candida species, although the difference was not statistically significant. This is similar to the results of previous studies which found that females, in general, have higher oral Candida colonization rates than males ${ }^{17,18}$ perhaps due to latent alteration of iron and vitamin B12 with pregnancies and hormonal changes. ${ }^{18-20}$
Previous studies reported that smoking significantly increased oral Candidal carriage rates, ${ }^{3,21}$ while others have failed to show a positive correlation between smoking and oral Candidal colonization. ${ }^{19}$ Alkumru and Beydermir ${ }^{22}$ demonstrated that cigarette smoking is an important factor that increased the Candidal growth in removable partial and complete denture wearers. Darwazeh et al. ${ }^{9}$ in a recent study found a significant correlation between the number of cigarettes smoked and the density of Candida isolates in oral rinse cultures. The present study showed a higher proportion of Candida carriers among smokers though not significantly different from non-smokers. This could be related to the small number of smoking participants in our study sample. In general, the results of this study found that Candida species were the most isolated species from the oral rinse samples.

At the initial visit and before the insertion of the prosthesis; of the 
50 subjects investigated, $48.0 \%$ yielded Candida in the oral culture and $64 \%$ of them were partially edentulous. Previous studies have shown an oral Candida carriage rate of $20-40 \%$ in Europe and North America, ${ }^{23,24} 53 \%$ in South Africa ${ }^{25}$ and $43 \%$ in the Middle East ${ }^{26}$ The prevalence reported in the present study falls within this range. Candida species have the ability to colonize dental plaque $e^{3,26}$ and is often found in dental carious lesions. ${ }^{27}$ This could explain the higher prevalence of oral Candida isolation in PDW's reported in this study as good oral hygiene was not one of the inclusion criteria.

At the first and second visits and after the insertion of the prosthesis at one and two months, the prevalence of oral Candida isolation increased from $48 \%$ to $50 \%$ and $54 \%$ respectively. Removable complete or partial dentures have shown to encourage the presence and growth of Candida, thus increasing the oral carriage rate ${ }^{20}$ which was the finding of other studies. ${ }^{28}$

Our analysis showed that ten different Candida species were isolated from the cultures of the oral rinse samples and three Candida species were isolated from the cultures of the fingertips samples. Candidaalbicans was the dominant yeast species among the subjects who yielded Candida in their oral cultures during the three visits; however, other Candida species were isolated too. It was reported in the literature that C. albicans occurs in about $30 \%-45 \%$ of healthy adults ${ }^{3,29,30}$ and $50-65 \%$ of people wearing removable dentures. ${ }^{3}$ The findings that $42 \%$ and $41-56 \%$ of subjects in this study were carriers of C. albicans at the initial vs. the first and second visits are similar to the results of the previous studies.

Darwazeh et al. ${ }^{8}$ have found that the hands of denture wearers who had oral Candida were significantly more colonized with Candida species than oral Candida-free subjects, suggesting the possibility of their hands contamination with the oral microbes during denture handling. This finding is contrary to the findings of the results in this study.

None of the subjects in this study were having concomitant positive oral and fingertips Candida speciescoincident at the same time at the initial visit and just three and two subjects at the first and second visits were having positive oral and fingertips Candida species respectively, which was statistically insignificant. However, the subjects included in that study ${ }^{8}$ were wearing removable dentures for at least 1 year, and about $56 \%$ of them were diagnosed with Candida-induced denture stomatitis. Moreover, they reported that subjects with denture-stomatitis were found to have a higher rate of fingertip Candida isolation (50\%) compared with those who did not have denture related stomatitis (36.4\%). In the present study, the subjects were wearing the prostheses for the first time and the samples were taken after just one and two months after the insertion. In addition, none of them have developed denture related stomatitis during this study period. The aforementioned findings may help explain the insignificant simultaneous oral and fingertips Candida isolation found. The absence of positive oral and fingertips Candida species carriers at the initial visit may highlight the role of denture handling in transmitting these microbes to the hands, but a longer study period is needed to investigate this.

Two subjects at the initial visit (one female CDW \& one female PDW) and another two (male \& female CDW's) at the second visit were found to have positive fingertips Candida but with negative oral isolations. One of the CDWs at the second visit was the same one at the initial visit and was a 70 year old female. The lower part of the gastrointestinal tract could be a source of Candida, especially in poor self-hygiene situations. ${ }^{8}$ Moreover, Candida species have been isolated from under the fingernails of women with vaginal candidiasis. ${ }^{30}$ Although subjects with skin fungal infections were excluded from this study, this was dependent on the medical history taken which was not confirmed by an expert clinical examination and was reliant on the subject's knowledge and reliability. Animal or environmental sources were also possible. ${ }^{19}$

The results of the current study have found no significant relationship between fingertips Candida carriage and hand washing. This is in accordance with the results of the Darwazeh et al. ${ }^{9}$ study and could be attributed to the relatively small size sample.

In the present Study, there was a significant correlation between not washing hands and increased oral Candida carriage. The clinical significance of candidal finger contamination should be determined, and the possibility that contaminated fingers will act as a reservoir of infection and reinfect the oral cavity that has been treated with antifungal therapy should be addressed. A recent study showed that the hands of $29 \%$ of hospital personnel were contaminated with Candida species; the species were not acquired from a common source. ${ }^{31}$ The failure of patient care providers in intensive care units to wash their hands has been linked to the hand carriage of strains of Candida isolated from the bloodstream of patients in these units. ${ }^{32}$

Plaque accumulation on the denture surface may create a suitable environment for Candida growth and infection. ${ }^{4}$ This study revealed a strong association between denture plaque and oral Candida and fungal non-Candida species colonization and growth. However, the relationship of density of Candidagrowth in saliva to the frequency of denture cleaning was not statistically significant. These findings are in agreement with the findings of other studies. ${ }^{4,8}$ Although the research has reached its aims, there were unavoidable limitations. First, because of the time limit, this research was conducted only in small size population. Therefore, to generalize the results for larger groups, the study should have involved more participants. Second, because of the subjects' awareness about the aims of this study; most of them had given more attention to their hands and dentures cleanliness, and this could have an impact on the results we gain. Finally, Phenotyping has certain limitation in the study of the epidemiology of human Candidal colonization and infection. Therefore, genotyping methods should be considered to investigate the epidemiology of Candida species in future studies. It is recommended that a larger sample of subjects should be investigated in future studies in longer follow up periods to precisely clarify the relationship between oral and fingertips Candida colonization. Further research identifying the sources of hand contamination is needed.

\section{Conclusion}

Within the limitations of this study,
the following conclusions can be drawn: The prevalence of oral Candidal carriage and the density of Candidal colonization were both higher after one and two months of the insertion of removable complete and partial dentures but the difference was not statistically significant.

1. There is no association between oral and fingertips Candida species presence among CDWs and PDWs. Candida species were isolated more frequently from the subjects' oral cavities with Candida albicans being the most isolated species.

2. A strong association was found between denture plaque and oral species colonization and growth. 


\section{Acknowledgment}

This study has been funded by Deanship of Scientific Research at Jordan University of Science and Technology (Grant 27/2011).

\section{Conflict of interest}

All authors of the manuscript titled Isolation of Candida Species from the Oral Cavity and Fingertips of Complete and Partial Dentures Wearers disclose any financial and personal relationships with other people or organizations that could inappropriately influence (bias) of this work.

\section{References}

1. Samaranayake LP. Host Factors and Oral Candidosis. In: Samaranayake LP, MacFarlane TW, editor. Oral Candidosis. Wright Butterworth, London;1990:66-103.

2. Odds FC. A Review and Bibliography. Candida and candidosis. Bailliere Tindall, London; $1988 ; 42-59$.

3. Arendorf TM, Walker DM. The prevalence and intra-oral distribution of Candida albicans in man. Arch Oral Biol. 1980;925(1):1-10.

4. Abaci O, Haliki-Uztan A, Ozturk B, et al. Determining Candida spp. incidence in denture wearers. Mycopathologia. 2010;169(5):365-372.

5. Scully C, el-Kabir M, Samaranayake LP. Candida and oral candidosis: a review. Crit Rev Oral Biol Med. 1994;5(2):125-157.

6. Yildirim M, Sahin I, Kucukbayrak A, et al. Hand carriage of Candida speciesand risk factors in hospital personnel. Mycoses. 2007;50(3):189-192.

7. Ruhnke M, Grosch-Worner I, Lischewski A, et al. Genotypic relatedness of yeast isolates from women infected with human immunodeficiency virus and their children. Mycoses. 1999;42(5-6):385-394.

8. Darwazeh AM, Al-Refai S, Al-Mojaiwel S. Isolation of Candida species from the oral cavity and fingertips of complete denture wearers. J Prosthet Dent. 2001;86(4):420-423.

9. Darwazeh AM, Al-Dwairi ZN, Al-Zwairi AA. The relationship between tobacco smoking and oral colonization with Candida species. J Contemp Dent Pract. 2010;11(3):17-24.

10. Williamson JJ. A study of extent of variation in daily counts of Candida albicans in saliva. Aust Dent J. 1972;17(2):106-109.

11. Samaranayake LP, MacFarlane TW, Lamey PJ, et al. A comparison of oral rinse and imprint sampling techniques for the detection of yeast, coliform and Staphylococcus aureus carriage in the oral cavity. J Oral Pathol. 1986;15(7):386-388.

12. Ambjornsen E, Rise J, Haugejorden O. A study of examiner errors associated with measurement of denture plaque. Acta Odontol Scand. 1984;42(3):183-191.

13. Graf B, Adam T, Zill E, et al. Evaluation of the VITEK 2 system for rapid identification of yeasts and yeast-like organisms. J Clin Microbiol. 2000;38(5):1782-1785.

14. Budtz-Jorgensen E. The significance of Candida albicans in denture stomatitis. Scand J Dent Res. 1974;82(2):151-190.
15. Paillaud E, Merlier I, Dupeyron C, et al. Oral candidiasis and nutritional deficiencies in elderly hospitalised patients. $\mathrm{Br} J \mathrm{Nutr}$. 2004;92(5):861-867.

16. Yuen HK, Wolf BJ, Bandyopadhyay D, et al. Oral health knowledge and behavior among adults with diabetes. Diabetes Res Clin Pract. 2009;86(3):239-246.

17. Zaremba ML, Daniluk T, Rozkiewicz D, et al. Incidence rate of Candidaspecies in the oral cavity of middle-aged and elderly subjects. Adv Med Sci. 2006;51(Suppl 1):233-236.

18. Al-Dwairi ZN. Complete edentulism and socioeconomic factors in a Jordanian population. Int J Prosthodont. 2010;23(6):541-543.

19. Rasool S, Siar CH, Ng KP. Oral Candidal species among smokers and non-smokers. J Coll Physicians Surg Pak. 2005;15(11):679-682.

20. Figueiral MH, Azul A, Pinto E, et al. Denture-related stomatitis: identification of aetiological and predisposing factors - a large cohort. $J$ Oral Rehabil. 2007;34(6):448-455.

21. Shin ES, Chung SC, Kim YK, et al. The relationship between oral Candida carriage and the secretor status of blood group antigens in saliva. Oral Surg Oral Med Oral Pathol Oral Radiol Endod. 2003;96(1):48-53.

22. Alkumru HN, Beydemir K. The prevalence of Candida albicans in complete denture and removable partial denture wearers: a comparative study. J Marmara Univ Dent Fac. 1992;1(3):218-222.

23. Hazen KC. New and emerging yeast pathogens. Clin Microbiol Rev. $1995 ; 8(4): 462-478$.

24. Kleinegger CL, Lockhart SR, Vargas K, et al. Frequency, intensity, species, and strains of oral Candidavary as a function of host age. J Clin Microbiol. 1996;34(9):2246-2254.

25. Masipa JN, Hauman $\mathrm{CH}$, Raubenheimer EJ. Oral carriage of Candida species in patients visiting the Medunsa Dental Clinic. J Dent Assoc S Afr. 1992;47(9):407-409.

26. Ellepola AN, Khan ZU, Joseph B, et al. Prevalence of Candida dubliniensis among oral Candida isolates in patients attending the Kuwait University Dental Clinic. Med Princ Pract. 2011;20(3):271-276.

27. Raja M, Hannan A, Ali K. Association of oral Candidal carriage with dental caries in children. Caries Res. 2010;44(3):272-276.

28. Ikebe K, Morii K, Matsuda K, et al. Association of Candidal activity with denture use and salivary flow in symptom-free adults over 60 years. $J$ Oral Rehabil. 2006;33(1):36-42.

29. Akpan A, Morgan R. Oral candidiasis. Postgrad Med J. 2002;78(922):455-459.

30. Riley VC, Odds FC, Fisk PE, et al. Finger nails as a reservoir for Candida albicans in recurrence of vaginal candidosis. Genitourin Med. 1986;62(5):358.

31. Huang YC, Lin TY, Leu HS, et al. Yeast carriage on hands of hospital personnel working in intensive care units. J Hosp Infect. 1998;39(1):47-51.

32. Pfaller MA. Epidemiology and control of fungal infections. Clin Infect Dis. 1994;19(Suppl 1):S8-S13. 